\title{
Well-Founded Extensive Games with Perfect Information
}

\author{
Krzysztof R. Apt \\ Centrum Wiskunde \& Informatica \\ Amsterdam, The Netherlands \\ University of Warsaw \\ Warsaw, Poland \\ k.r.apt@cwi.nl
}

\author{
Sunil Simon \\ Department of CSE, \\ IIT Kanpur, Kanpur, India \\ simon@cse.iitk.ac.in
}

We consider extensive games with perfect information with well-founded game trees and study the problems of existence and of characterization of the sets of subgame perfect equilibria in these games. We also provide such characterizations for two classes of these games in which subgame perfect equilibria exist: two-player zero-sum games with, respectively, two and three outcomes.

\section{Introduction}

Research on strategic games assumes that players have to their disposal infinitely many strategies. This allows one to view strategic games with mixed strategies as customary strategic games with infinitely many strategies. This assumption is also used in a study of various standard examples, such as Cournot or Bertrand competition, in which the players have to set the production level or the price of a product.

In contrast, the exposition of the standard results for the extensive games with perfect information (from now, just 'extensive games') is usually limited to finite games. This restriction rules out a study of various natural examples, for example infinite variants of the ultimatum game or some bargaining games, see, e.g., [16]. In the first case the game has just two stages but the first player may have infinitely many actions to choose from, while in the second case the game has an arbitrary, though finite, number of stages. Such games are then analyzed separately, without taking into account general results.

The aim of this paper is to provide a systematic account of extensive games with perfect information in a setting that only requires that the underlying game tree is well-founded (i.e., has no infinite paths). We call such games well-founded.1.

The standard tool to analyze finite extensive games is the concept of a subgame perfect equilibrium. Their existence is established by means of the backward induction algorithm. In infinite well-founded extensive games subgame perfect equilibria may fail to exist. Also one cannot resort to any version of this algorithm since it will not terminate. In principle this could be taken care of by defining a joint strategy as an eventual outcome of an infinite computation. However, for arbitrary well-founded games one would have then to proceed by means of a transfinite induction, which raises a legitimate question whether such a process can be called a computation.

Therefore, instead of trying to define such generalized computations we dispense with the backward induction altogether and simply proceed by transfinite induction. This results in mathematical proofs of existence that are not supported by any algorithm, but still, as illustrated by examples, the obtained results can be used to compute the sets of subgame perfect equilibria in specific well-founded games and to deduce their existence in special cases. Informally, transfinite induction analyzes the game tree 'top

\footnotetext{
${ }^{1}$ Such games are sometimes called games with finite horizon (see, e.g., [15]). We decided to use instead the qualification 'well-founded' because 'finite horizon' is sometimes used to indicate that the game tree is of bounded depth, i.e, has a finite rank (a concept introduced in the next section).
}

J. Y. Halpern and A. Perea (Eds.): Theoretical Aspects of Rationality and Knowledge 2021 (TARK 2021)

EPTCS 335, 2021, pp. 7-21 doi 10.4204/EPTCS.335.2
(C) K. R. Apt \& S. Simon

This work is licensed under the Creative Commons Attribution License. 
down', while the backward induction proceeds 'bottom up' and consequently cannot be naturally applied to infinite game trees.

Most results, though not all, are natural generalizations of the corresponding results for the case of finite extensive games. Some of these results fail to hold for infinite games and some of the traditional proofs for finite games, notably the ones involving the backward induction, have to be suitably modified. In what follows we focus both on arbitrary well-founded games and on two-player zero-sum games with, respectively, two and three outcomes.

In the literature we found only one paper in which well-founded games appear, namely [5]. The authors provide using higher-order computability theory a formula that defines the set of subgame perfect equilibria under an assumption that implies their existence, and apply it to determine a subgame perfect equilibrium in an infinite three stage game. In several books various examples of infinite extensive games are studied and various extensions of finite extensive games, for example games with chance moves, see, e.g., [16], simultaneous moves, see, e.g., [15], or repeated extensive games, see [13], are introduced (not to mention games with imperfect information). Also subgame perfect equilibria in games allowing infinite plays have been studied, see, e.g., [8] and a more recent [10]. In [1] a maximally general definition of an extensive form game is proposed that among others covers repeated games, differential games, and stochastic games. In the proposed framework even immediate predecessors of an action may not exist (like in continuous time interactive decisions examples). In our opinion the class of games considered here merits attention as a first natural generalization to study.

In the next section we introduce the relevant concepts and provide natural examples of well-founded extensive games. In Section 3 we establish existence of subgame perfect equilibria for some natural classes of well-founded games and show how to apply a characterization result to compute the set of subgame perfect equilibria for specific example games. Then, in Section 4 we consider two classes of two-player well-founded games: win or lose games and chess-like games. As a stepping stone towards characterizations of the sets of subgame perfect equilibria in these games we show that the well-known result attributed to Zermelo [23] (see also [19]) about existence of winning strategies continues to hold for well-founded games.

\section{Preliminaries on extensive games}

A tree is an acyclic directed connected graph, written as $(V, E)$, where $V$ is a non-empty set of nodes and $E$ is a possibly empty set of edges. In drawings the edges will be directed downwards.

An extensive game with perfect information (in short, just an extensive game) for $n \geq 1$ players consists of:

- a set of players $\{1, \ldots, n\}$,

- a game tree, which is a tree $T:=(V, E)$ with a turn function turn $: V \backslash Z \rightarrow\{1, \ldots, n\}$, where $Z$ is the set of leaves of $T$,

- the payoff functions $p_{i}: Z \rightarrow \mathbb{R}$, for each player $i$.

We denote it by $\left(T\right.$, turn $\left., p_{1}, \ldots, p_{n}\right)$.

The function turn determines at each non-leaf node which player should move. The edges of $T$ represent possible moves in the considered game, while for a node $v \in V \backslash Z$ the set of its children $C(v):=\{w \mid(v, w) \in E\}$ represents possible actions of player turn $(v)$ at $v$. For a node $u$ in $T$ let $T^{u}$ denote the subtree of $T$ rooted at $u$. 
We say that an extensive game is finite, finite depth, infinite, or well-founded if, respectively, its game tree is finite, finite depth, infinite, or well-founded. Recall that a tree is called well-founded if it has no infinite paths (see, e.g., [21, page 224]).

Further, following [2], we say that an extensive game is without relevant ties if for all non-leaf nodes $u$ in $T$ the function $p_{i}$, where $\operatorname{turn}(u)=i$, is injective on the leaves of $T^{u}$. This is more general than saying that a game is generic, which means that each $p_{i}$ is an injective function.

We shall often rely on the concept of a rank of a well-founded tree $T$. Recall that it is defined inductively as follows, where $v$ is the root of $T$ :

$$
\operatorname{rank}(T):= \begin{cases}0 & \text { if } T \text { has one node } \\ \sup \left\{\operatorname{rank}\left(T^{u}\right)+1 \mid u \in C(v)\right\} & \text { otherwise, }\end{cases}
$$

where $\sup (X)$ denotes the least ordinal larger than all ordinals in the set $X$. Transfinite induction will be needed only to deal with games on the trees with rank $>\omega$.

In the figures below we identify the actions with the labels we put on the edges and thus identify each action with the corresponding move. For convenience we do not assume the labels to be unique, but it will not lead to confusion. Further, we annotate the non-leaf nodes with the identity of the player whose turn it is to move and the name of the node. Finally, we annotate each leaf node with the corresponding sequence of the values of the $p_{i}$ functions.

Example 1 The following two-player game is called the Ultimatum game. Player 1 moves first and claims a real number $x \in[0,100]$, to be interpreted as a fraction of some good to be shared, leaving the fraction $100-x$ for the other player. Player 2 either accepts this decision, the outcome is then $(x, 100-x)$, or rejects it, the outcome is then $(0,0)$. The game tree is depicted in Figure 1, where the action of player 1 is a number from the set $[0,100]$, and the actions of player 2 are denoted by $A$ and $R$. The resulting game is infinite but is of finite depth. The rank of the game tree is 2 .

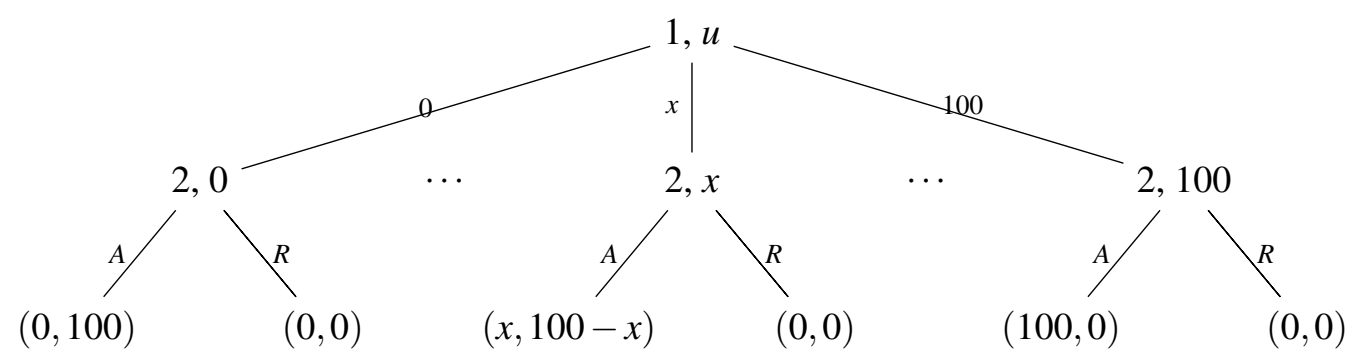

Figure 1: The Ultimatum game

Example 2 Consider the following Bargaining game (without depreciation). Player 1 moves first by selecting a natural number $k \geq 2$. Such a choice is to be interpreted that he claims the fraction $1-\frac{1}{k}$ of some good to be shared, leaving the fraction $\frac{1}{k}$ for the other player. As long as player 1 selects $k>2$, player 2 asks for a better offer or rejects it. In the first case player 1 selects $k-1$. The game continues until player 1 selects 2 , i.e., claims $50 \%$ of the good. At that moment player 2 either accepts this offer, the outcome is then $(50,50)$, or rejects it. All rejections result in the outcome $(0,0)$. The game tree of this game, depicted in Figure 2, has arbitrary long, though finite, branches, so this game is infinite but it is well-founded. The rank of the game tree is $\omega$. 


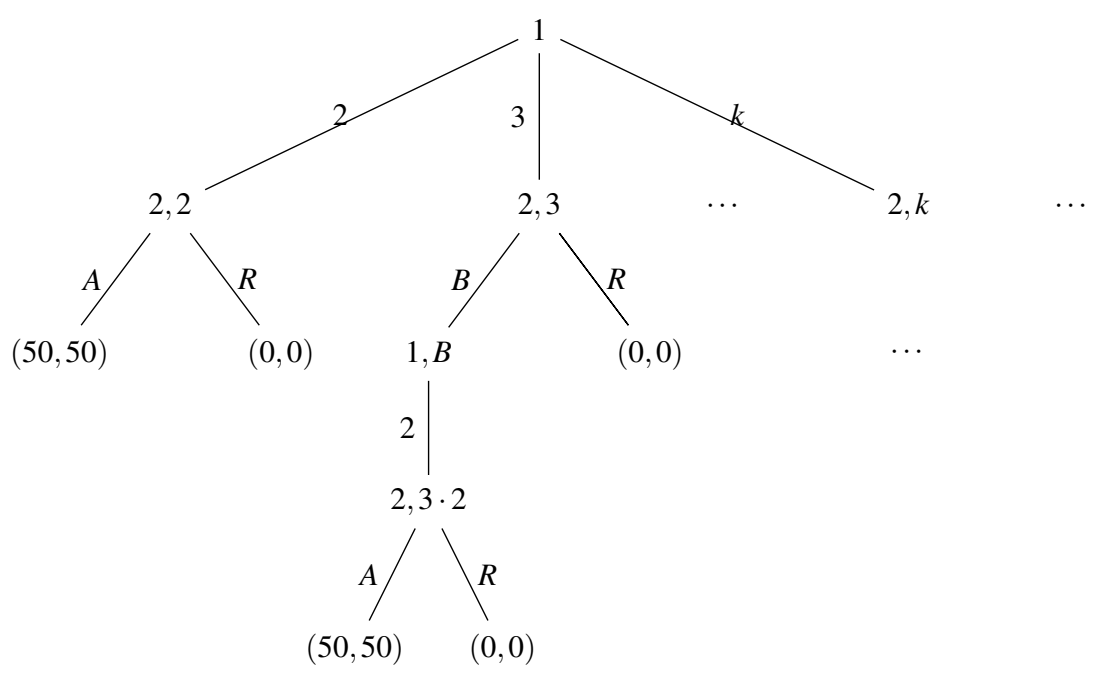

Figure 2: The Bargaining game

Below, given a two-player extensive game we denote the opponent of player $i$ by $-i$ instead of $3-i$.

Example 3 We now construct a sequence of games $G(i, \alpha)$, where $i \in\{1,2\}$ and $\alpha$ is an ordinal $>1$, by induction as follows:

- $G(1,2)$ is the the ultimatum game from Example 1 and $G(2,2)$ is its version with the roles of players 1 and 2 reversed;

- $G(i, \alpha)$, where $\alpha>2$, is obtained as follows:

- its game tree is constructed by selecting a root $v$, taking the game trees of the games $G(-i, \beta)$, where $1<\beta<\alpha$ and selecting their roots as the children of $v$,

- setting $\operatorname{turn}(v)=i$.

So for example the root of the game tree of $G(i, 3)$ has one child, namely the root of the game tree of $G(-i, 2)$, the root of the game tree of $G(i, 4)$ has two children, namely the roots of the game trees of $G(-i, 3)$ and $G(-i, 2)$, etc. Note that the rank of the game tree of $G(i, \alpha)$ is $\alpha$.

The class of endogenous games studied in [9] form another example of well-founded extensive games. These games are played in two stages. In the first stage the players are involved in pre-play negotiations that essentially fix the payoff functions and in the second stage they choose their strategies. The resulting game is infinite due to the pre-play negotiations, while the rank of the game tree is 2.

Note that by König's lemma [11] every finitely branching well-founded extensive games is finite. Consequently, interesting well-founded extensive games necessarily have infinite branching.

For an extensive game $G:=\left(T, \operatorname{turn}, p_{1}, \ldots, p_{n}\right)$ let $V_{i}:=\{v \in V \backslash Z \mid \operatorname{turn}(v)=i\}$. So $V_{i}$ is the set of nodes at which player $i$ moves. A strategy for player $i$ is a function $s_{i}: V_{i} \rightarrow V$, such that $\left(v, s_{i}(v)\right) \in E$ for all $v \in V_{i}$. We denote the set of strategies of player $i$ by $S_{i}$.

Let $S=S_{1} \times \cdots \times S_{n}$. We call each element $s \in S$ a joint strategy, denote the $i$ th element of $s$ by $s_{i}$, and abbreviate the sequence $\left(s_{j}\right)_{j \neq i}$ to $s_{-i}$. We write $\left(s_{i}^{\prime}, s_{-i}\right)$ to denote the joint strategy in which player $i$ 's strategy is $s_{i}^{\prime}$ and for all $j \neq i$, player $j$ 's strategy is $s_{j}$. Occasionally we write $\left(s_{i}, s_{-i}\right)$ instead of $s$. Finally, we abbreviate the Cartesian product $\times_{j \neq i} S_{j}$ to $S_{-i}$. So in the degenerate situation when the game 
tree consists of just one node, each strategy is the empty function, denoted by $\emptyset$, and there is only one joint strategy, namely the $n$-tuple of these functions. Each joint strategy assigns a unique descendant to every node in $V \backslash Z$. In fact, we can identify joint strategies with such assignments.

From now on the above notation will be used in the context of any considered extensive game $G$. In particular $S_{i}$ will always denote the set of strategies of player $i$.

Each joint strategy $s=\left(s_{1}, \ldots, s_{n}\right)$ determines a rooted path play $(s):=\left(v_{1}, \ldots, v_{m}\right)$ in $T$ defined inductively as follows:

- $v_{1}$ is the root of $T$,

- if $v_{k} \notin Z$, then $v_{k+1}:=s_{i}\left(v_{k}\right)$, where $\operatorname{turn}\left(v_{k}\right)=i$.

So when the game tree consists of just one node, $v$, we have play $(s)=v$. Informally, given a joint strategy $s$, we can view play $(s)$ as the resulting play of the game.

Suppose now that the extensive game is well-founded. Then for each joint strategy $s$ the rooted path $\operatorname{play}(s)$ is finite. Denote by leaf $(s)$ the last element of play $(s)$. We call $\left(p_{1}(\right.$ lea $f(s)), \ldots, p_{n}($ leaf $\left.(s))\right)$ the outcome of the game $G$ when each player $i$ pursues his strategy $s_{i}$ and abbreviate it as $p($ lea $f(s))$. We call two joint strategies $s$ and $t$ payoff equivalent if $p($ leaf $(s))=p($ lea $f(t))$.

We say that a strategy $s_{i}$ of player $i$ a best response to a joint strategy $s_{-i}$ of his opponents if for all $s_{i}^{\prime} \in S_{i}, p_{i}($ leaf $(s)) \geq p_{i}\left(\right.$ leaf $\left.\left(s_{i}^{\prime}, s_{-i}\right)\right)$. Next, we call a joint strategy $s$ a Nash equilibrium if each $s_{i}$ is a best response to $s_{-i}$, that is, if

$$
\forall i \in\{1, \ldots, n\}, \forall s_{i}^{\prime} \in S_{i}, p_{i}\left(\operatorname{leaf}\left(s_{i}, s_{-i}\right)\right) \geq p_{i}\left(\operatorname{leaf}\left(s_{i}^{\prime}, s_{-i}\right)\right) .
$$

Example 4 Let us return to the Ultimatum game from Example 1. Each strategy for player 1 is a number, respectively from $[0,100]$, while each strategy for player 2 assigns to every such number $x$ either $A$ or $R$.

It is easy to check that each Nash equilibrium is of the form $(100$, always $R)$, with the outcome $(100,0)$, or $\left(x, s_{2}\right)$ with $s_{2}(x)=A$ and $s_{2}(y)=R$ for $y>x$, where $x, y \in[0,100]$, with the outcome $(x, 100-x)$.

Finally, we recall the notion of a subgame perfect equilibrium due to Selten [20](see also section 6.2 in [15]), though now defined for the larger class of well-founded games.

The subgame of G rooted at the node $w$, denoted by $G^{w}$, is defined as follows:

- its set of players is $\{1, \ldots, n\}$,

- its tree is $T^{w}$,

- its turn and payoff functions are the restrictions of the corresponding functions of $G$ to the nodes of $T^{w}$.

Note that some players may 'drop out' in $G^{w}$, in the sense that at no node of $T^{w}$ it is their turn to move. Still, to keep the notation simple, it is convenient to admit in $G^{w}$ all original players in $G$.

Each strategy $s_{i}$ of player $i$ in $G$ uniquely determines his strategy $s_{i}^{w}$ in $G^{w}$. Given a joint strategy $s=\left(s_{1}, \ldots, s_{n}\right)$ of $G$ we denote by $s^{w}$ the joint strategy $\left(s_{1}^{w}, \ldots, s_{n}^{w}\right)$ in $G^{w}$. Further, we denote by $S_{i}^{w}$ the set of strategies of player $i$ in the subgame $G^{w}$ and by $S^{w}$ the set of joint strategies in this subgame.

Suppose now the extensive game $G$ is well-founded. Then the notion of a Nash equilibrium is welldefined. A joint strategy $s$ of $G$ is called a subgame perfect equilibrium in $G$ if for each node $w$ of $T$, the joint strategy $s^{w}$ of $G^{w}$ is a Nash equilibrium in $G^{w}$. Informally $s$ is subgame perfect equilibrium in $G$ if it induces a Nash equilibrium in every subgame of $G$. 


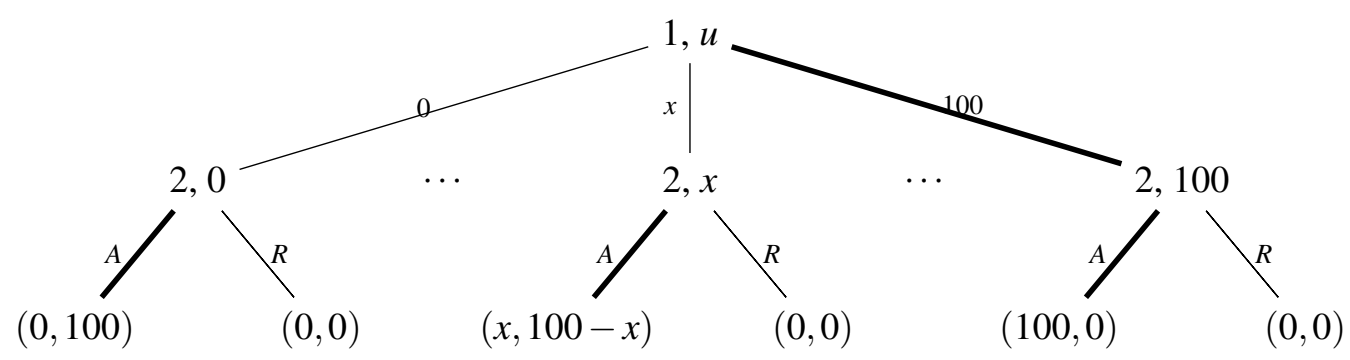

Figure 3: The subgame perfect equilibrium in the Ultimatum game

Example 5 Return now to the Ultimatum game from Example 1. It is easy to check that it has exactly one subgame equilibrium, depicted in Figure 3 by thick lines.

Note that even though the rank of the game tree is 2, the customary backward induction cannot be applied here to compute this subgame equilibrium. Indeed, to deal with the root node the algorithm has to deal first with infinitely many nodes at which player 2 moves, which leads to divergence.

An analysis of the subgame perfect equilibria in the games from Examples 2 and 3 is more involved and will be provided in the next section using a characterization of the set of subgame perfect equilibria of a well-founded extensive game.

\section{Subgame perfect equilibria in well-founded games}

In general subgame perfect equilibria may not exist in well-founded games. As an example take the modification of the Ultimatum game from Example 1 in which instead of $[0,100]$ one considers the open interval $(0,100)$. In this section we establish existence of subgame perfect equilibria in some natural classes of well-founded games. This will directly follow from a characterization of the sets of subgame perfect equilibria in such games.

We begin by stating a preparatory lemma, called the 'one deviation property' in [15]. To keep the paper self-contained we include in the Appendix the proof. It is more detailed than the one given in [15].

Lemma 6 Let $G$ be a well-founded extensive game over the game tree T. A joint strategy $s$ is a subgame perfect equilibrium in $G$ iff for all non-leaf nodes $u$ in $T$ and all $y \in C(u)$

- $p_{i}\left(\operatorname{leaf}\left(s^{x}\right)\right) \geq p_{i}\left(\right.$ leaf $\left.\left(s^{y}\right)\right)$, where $i=\operatorname{turn}(u)$ and $s_{i}(u)=x$.

Corollary 7 Let $G$ be a well-founded extensive game over the game tree $T$ with the root $v$. A joint strategy $s$ is a subgame perfect equilibrium in $G$ iff for all $u \in C(v)$

- $p_{i}\left(\right.$ leaf $\left.\left(s^{w}\right)\right) \geq p_{i}\left(\right.$ leaf $\left.\left(s^{u}\right)\right)$, where $i=\operatorname{turn}(v)$ and $s_{i}(v)=w$,

- $s^{u}$ is a subgame perfect equilibrium in the subgame $G^{u}$.

Intuitively, the first condition states that among the subgames rooted at the children of the root $v$, the one determined by the first move in the game $G$ yields the maximal outcome for the player who moved. Recall that for a function $f: X \rightarrow Y$ (with $X$ non-empty), $\operatorname{argmax}_{x \in X} f(x):=\left\{y \in X \mid f(y)=\max _{x \in X} f(x)\right\}$. Using this notation this condition can be reformulated as: $s_{i}(v) \in \operatorname{argmax}_{u \in C(v)} p_{i}\left(\right.$ leaf $\left.\left(s^{u}\right)\right)$, where $i=\operatorname{turn}(v)$. 
Proof. If $C(v)=\emptyset$, the claim is vacuously true. Otherwise consider any $u \in C(v)$. By Lemma $6 s^{u}$ is a subgame perfect equilibrium in $G^{u}$ iff for all non-leaf nodes $y$ in $T^{u}$ and $z \in C(y), p_{i}\left(\right.$ leaf $\left(\left(s^{u}\right)^{x}\right) \geq$ $p_{i}\left(\right.$ leaf $\left(\left(s^{u}\right)^{z}\right)$, where $i=\operatorname{turn}(y)$ and $s_{i}^{u}(y)=x$.

Since $\left(s^{u}\right)^{x}=s^{x}$ and $\left(s^{u}\right)^{z}=s^{z}$, the last statement is equivalent to the statement that the inequality in Lemma 6 holds for all non-leaf nodes $y$ in $T^{u}$ and $z \in C(y)$. The conclusion now follows by Lemma 6 .

The above corollary allows us to characterize inductively the set of subgame perfect equilibria in each well-founded extensive game.

Consider a well-founded extensive game $G$ with the root $v$ and suppose $C(v) \neq \emptyset$. Consider the subgames $G^{w}$, where $w \in C(v)$, and a function $f$ that assigns to each sequence $t$ of joint strategies in these subgames a child of $v$. Then each pair of $t$ and $f$ determines a joint strategy in $G$ that we denote by $(f, t)$.

Recall that by $S^{w}$ we denote the set of joint strategies in the subgame $G^{w}$. Given subsets $U^{w}$ of $S^{w}$ for $w \in C(v)$ and a set of functions $F$ from $\times_{w \in C(v)} U^{w}$ to $C(v)$, we denote by $\left[F, \times_{w \in C(v)} U^{w}\right]$ the set of joint strategies in $G$ defined by

$$
\left[F, \times_{w \in C(v)} U^{w}\right]:= \begin{cases}\{(\emptyset, \ldots, \emptyset)\} & \text { if } C(v)=\emptyset \\ \left\{(f, t) \mid f \in F, t \in \times_{w \in C(v)} U^{w}\right\} & \text { otherwise }\end{cases}
$$

In the first case $(\emptyset, \ldots, \emptyset)$ stands for the joint strategy that consists of the $n$-tuple of the empty strategies. Note that when $C(v) \neq \emptyset$ if any of the sets $U^{w}$ or $F$ is empty, then so is $\left[F, \times_{w \in C(v)} U^{w}\right]$. Further, we denote the set of subgame perfect equilibria in $G$ by $\operatorname{SPE}(G)$.

Theorem 8 Consider a well-founded extensive game $G$ with the root $v$ and let $i=\operatorname{turn}(v)$. Then

$$
\operatorname{SPE}(G)=\left[F, \times_{w \in C(v)} \operatorname{SPE}\left(G^{w}\right)\right],
$$

where if $C(v) \neq \emptyset$ then $F=\left\{f \mid \forall t \in \times_{w \in C(v)} \operatorname{SPE}\left(G^{w}\right) f(t) \in \operatorname{argmax}_{w \in C(v)} p_{i}\left(\right.\right.$ leaf $\left.\left.\left(t^{w}\right)\right)\right\}$.

In particular, if the set $\operatorname{argmax}_{w \in C(v)} p_{i}\left(\right.$ leaf $\left.\left(t^{w}\right)\right)$ is empty, then $F=\emptyset$ and hence $S P E(G)=\emptyset$. Intuitively, each function $f \in F$, given a sequence of subgame perfect equilibria in the subgames rooted at the children of the root $v$, selects a root of the subgame in which the outcome in the equilibrium is maximal for the player who moves at $v$.

Proof of Theorem 8 . If $C(v)=\emptyset$, then $(\emptyset, \ldots, \emptyset)$ is a unique subgame perfect equilibrium, so the claim holds.

If $C(v) \neq \emptyset$, then by Corollary 7 every subgame perfect equilibrium in the game $G$ is of the form $(f, t)$, where for all $w \in C(v), t^{w}$ is a subgame perfect equilibrium in $G^{w}$ and for some $w \in C(v)$ we have $f(t)=w$ and $p_{i}\left(\operatorname{leaf}\left(s^{w}\right)\right) \geq p_{i}\left(\right.$ leaf $\left.\left(s^{u}\right)\right)$ for all $u \in C(v)$.

Corollary 9 Every well-founded extensive game with finitely many outcomes has a subgame perfect equilibrium.

Proof. The claim follows from Theorem 8 by induction on the rank of the game tree and the observation that for every function $g: X \rightarrow Y$ with a finite range the set $\operatorname{argmax}_{x \in X} g(x)$ is non-empty.

The above result can be generalized to some games with infinitely many outcomes. An example is a game in which for each player the set of outcomes is either finite or equals the set of negative integers. More generally, consider a well-founded extensive game in which for each player the set of outcomes is a reverse well-ordered set, i.e., every subset of this set has a greatest element. Then Theorem 8 implies that the game has a subgame perfect equilibrium.

Corollary 10 Every well-founded extensive game without relevant ties has at most one subgame perfect equilibrium. 
Proof. If a game is without relevant ties, then so is every subgame of it. This allows us to proceed by induction on the rank of the game tree. For game trees of rank 0 the claim clearly holds. Suppose that it holds for all well-founded extensive games without relevant ties with the game trees of rank smaller than some ordinal $\alpha>0$. Consider such a game with game tree of rank $\alpha$ and rooted at $v$. Let $i=\operatorname{turn}(v)$.

By the induction hypothesis for each $w \in C(v)$ the set $\operatorname{SPE}\left(G^{w}\right)$ has at most one element. If one of these sets is empty, then so is $\operatorname{SPE}(G)$.

So suppose that each $\operatorname{SPE}\left(G^{w}\right)$ is a singleton set. Then so is $\times_{w \in C(v)} \operatorname{SPE}\left(G^{w}\right)$. Let $\times_{w \in C(v)} \operatorname{SPE}\left(G^{w}\right)=$ $\{t\}$. Then for different $w, w^{\prime} \in C(v)$, leaf $\left(t^{w}\right)$ and leaf $\left(t^{w^{\prime}}\right)$ are different leaves of the game tree of $G$, so by the assumption about the game $p_{i}\left(\right.$ leaf $\left.\left(t^{w}\right)\right) \neq p_{i}\left(\right.$ leaf $\left.\left(t^{w^{\prime}}\right)\right)$, since $i=\operatorname{turn}(v)$.

This means that the function $g: C(v) \rightarrow \mathbb{R}$ defined by $g(w):=p_{i}\left(\right.$ leaf $\left.\left(t^{w}\right)\right)$ is injective. Consequently the set $\operatorname{argmax}_{w \in C(v)} p_{i}\left(\right.$ leaf $\left.\left(t^{w}\right)\right)$ has at most one element and hence the same successively holds for the sets $F$ and $\operatorname{SPE}(G)$.

In particular, every generic well-founded extensive game with finitely many outcomes has a unique subgame perfect equilibrium. We now show how Theorem 8 can be used to reason about subgame perfect equilibria in specific extensive games.

Example 11 Consider the Bargaining game $G$ from Example 2, Denote by $G(k)$ the game in which player 1 first selects the number $k$. The inductive structure of these games is depicted in Figure 4, where the actions of player 2 are $B$ ('make a better offer') or $A$ and $R$, as in Example 1 .
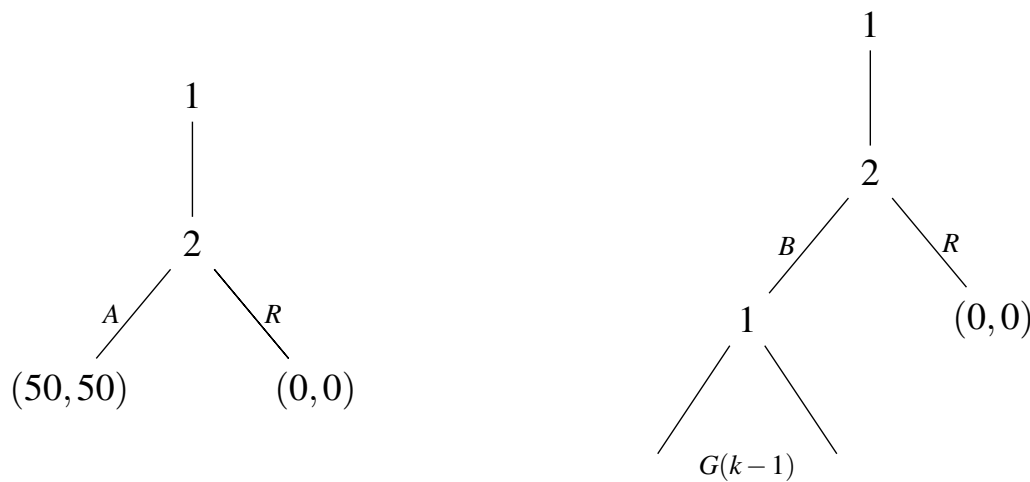

Figure 4: The games $G(2)$ and $G(k)$ for $k>2$

It is easy to prove by induction using Theorem 8 or simply by the backward induction (the presentation of which we omit) that each game $G(k)$, where $k \geq 2$, has a unique subgame perfect equilibrium with the outcome $(50,50)$.

Children of the root of the game tree of $G$ are the roots of the game trees of $G(k)$, where $k \geq 2$. So for the game $G$ the set $\operatorname{argmax}_{w \in C(v)} p_{i}\left(l e a f\left(t^{w}\right)\right)$ referred to in Theorem 8 has exactly one element, 50 . Hence Theorem 8 implies that $G$ has a subgame perfect equilibrium, that the outcome in each of them is $(50,50)$, and that for each $k \geq 2$ there is a unique subgame perfect equilibrium in which player 1 first selects $k$.

Example 12 Consider now the games $G(i, \alpha)$, where $i \in\{1,2\}$ and $\alpha$ is an ordinal $>1$ from Example 3 . We noticed in Example 5 that the game $G(1,2)$ has a unique subgame perfect equilibrium with the outcome $(100,0)$. By symmetry the game $G(2,2)$ has a unique subgame perfect equilibrium with the outcome $(0,100)$. The root of the game tree $G(i, 3)$ has one child, which is the root of the game tree of $G(-i, 2)$. Consequently $G(i, 3)$ has a unique subgame perfect equilibrium with the outcome $(0,100)$ for $i=1$ and $(100,0)$ for $i=2$. 
Using these observations we now show that for $i \in\{1,2\}$ and ordinals $\alpha>3$ the game $G(i, \alpha)$ has a subgame perfect equilibrium and the outcomes in all these equilibria are all $(100,0)$ for $i=1$ and $(0,100)$ for $i=2$. We proceed by induction. Consider a game $G(1, \alpha)$ with $\alpha>3$ and assume the claim holds for all $\beta$ with $3 \leq \beta<\alpha$. The root of the game tree has as children the roots of the game trees of $G(2, \beta)$, where $1<\beta<\alpha$.

By the induction hypothesis all these games except $G(2,3)$ have subgame perfect equilibria with the outcomes $(0,100)$. For the game $G(2,3)$, as just noted, the outcome in the unique subgame perfect equilibrium is $(100,0)$. So for the game $G(1, \alpha)$ the set $\operatorname{argmax}_{w \in C(v)} p_{i}\left(l e a f\left(t^{w}\right)\right)$ referred to in Theorem 8 has exactly one element, 100 . Using this theorem we conclude that the game $G(1, \alpha)$ has a subgame perfect equilibrium and that the outcome in each equilibrium is $(100,0)$. A symmetric claim, referring to $(0,100)$ instead, holds for each game $G(2, \alpha)$ with $\alpha>3$.

Using Theorem 8 we conclude that each $G(i, \alpha)$ for $\alpha>4$ has multiple subgame perfect equilibria.

Next, we establish a result showing that for a class of well-founded extensive games all subgame perfect equilibria are payoff equivalent. The following condition was introduced in [17]:

$$
\forall i \in\{1, \ldots, n\} \forall s, t \in S\left[p_{i}(\text { leaf }(s))=p_{i}(\text { leaf }(t)) \rightarrow p(\text { leaf }(s))=p(\text { leaf }(t))\right] .
$$

This condition is in particular satisfied by the two-player well-founded extensive games that are strictly competitive, which means that

$$
\forall i \in\{1,2\} \forall s, s^{\prime} \in S p_{i}(\text { leaf }(s)) \geq p_{i}\left(\text { leaf }\left(s^{\prime}\right)\right) \text { iff } p_{-i}(\text { leaf }(s)) \leq p_{-i}\left(\text { leaf }\left(s^{\prime}\right)\right) .
$$

(To see it transpose $i$ and $-i$ and conjoin both equivalences.)

Theorem 13 In every well-founded extensive game that satisfies condition (1) all subgame perfect equilibria are payoff equivalent.

Proof. First we prove the following claim.

Claim. If a well-founded extensive game satisfies condition (1), then so does every subgame of it.

Proof. Let $G$ be a well-founded extensive game that satisfies condition (1). Consider any subgame $G^{w}$ of $G$. Suppose that for some player $i$ and joint strategies $s^{\prime}$ and $t^{\prime}$ in $G^{w}$ we have $p_{i}\left(\right.$ leaf $\left.\left(s^{\prime}\right)\right)=p_{i}\left(\right.$ leaf $\left.\left(t^{\prime}\right)\right)$. Take some joint strategies $s$ and $t$ in $G$ such that leaf $(s)=$ leaf $\left(s^{\prime}\right)$, leaf $(t)=$ leaf $\left(t^{\prime}\right), s^{w}=s^{\prime}$ and $t^{w}=t^{\prime}$. Then $p_{i}($ leaf $(s))=p_{i}($ leaf $(t))$, so by condition (1) $p($ leaf $(s))=p($ leaf $(t))$ and consequently $p\left(\right.$ leaf $\left.\left(s^{\prime}\right)\right)=p\left(\right.$ leaf $\left.\left(t^{\prime}\right)\right)$.

We now proceed by induction on the rank of the game tree. For game trees of rank 0 the claim obviously holds. Suppose the claim holds for all well-founded extensive games whose game tree is of rank smaller than some ordinal $\alpha>0$. Consider a well-founded game $G=\left(T, t u r n, p_{1}, \ldots, p_{n}\right)$ over a game tree of rank $\alpha$ with the root $v$. Take two subgame perfect equilibria $s$ and $t$ in $G$.

If $\operatorname{path}(s)=\operatorname{path}(t)$, then $p($ leaf $(s))=p($ leaf $(t))$. Otherwise take the first non-leaf node $u$ lying on $\operatorname{path}(s)$ such that $s_{i}(u) \neq t_{i}(u)$, where $i=\operatorname{turn}(u)$. Let $s_{i}(u)=x$ and $t_{i}(u)=y$.

Both $s^{y}$ and $t^{y}$ are subgame perfect equilibria in the subgame $G^{y}$. By the Claim the game $G^{y}$ satisfies condition (1), so by the induction hypothesis $s^{y}$ and $t^{y}$ are payoff equivalent in $G^{y}$. We thus have

$$
p_{i}(\operatorname{leaf}(s))=p_{i}\left(\operatorname{leaf}\left(s^{x}\right)\right) \geq p_{i}\left(\operatorname{leaf}\left(s^{y}\right)\right)=p_{i}\left(\operatorname{leaf}\left(t^{y}\right)\right)=p_{i}(\operatorname{leaf}(t)),
$$

where the inequality holds by Lemma 6. Analogously $p_{i}($ leaf $(t)) \geq p_{i}($ leaf $(s))$, so $p_{i}($ leaf $(s))=$ $p_{i}($ leaf $(t))$ and hence by condition (1) $p($ leaf $(s))=p($ leaf $(t))$. 
For finite extensive games this result was stated in [15, page 100] as Exercise 100.2. The most natural proof makes use of the backward induction. For infinite games a different proof is needed.

We say that a well-founded extensive game $\left(T\right.$, turn $\left., p_{1}, \ldots, p_{n}\right)$ satisfies the transference of decisionmaker indifference (TDI) condition if: $\forall i \in\{1, \ldots, n\} \forall r_{i}, t_{i} \in S_{i} \forall s_{-i} \in S_{-i}$,

$$
p_{i}\left(\operatorname{leaf}\left(r_{i}, s_{-i}\right)\right)=p_{i}\left(\operatorname{leaf}\left(t_{i}, s_{-i}\right)\right) \rightarrow p\left(\operatorname{leaf}\left(r_{i}, s_{-i}\right)\right)=p\left(\operatorname{leaf}\left(t_{i}, s_{-i}\right)\right) .
$$

Informally, this condition states that whenever for some player $i$ two of his strategies $r_{i}$ and $t_{i}$ are indifferent w.r.t. some joint strategy $s_{-i}$ of the other players then this indifference extends to all players.

Clearly, condition (1) implies the TDI condition. The TDI condition was introduced in [14], the results of which imply that in every finite extensive game with perfect information that satisfies the TDI condition all subgame perfect equilibria are payoff equivalent. We conjecture that this result extends to well-founded extensive games.

\section{Win or lose and chess-like games}

In this section we characterize subgame perfect equilibria of two-player zero-sum well-founded extensive games with, respectively, two and three outcomes. By Corollary 9 each of these games has a subgame perfect equilibrium. Below we consider the outcomes $(1,-1),(0,0)$, and $(-1,1)$, but the obtained results hold with the same proofs for arbitrary outcomes as long as the game remains zero-sum.

A two-player extensive game is called a win or lose game if the only possible outcomes are $(1,-1)$ and $(-1,1)$, with 1 associated with winning and 0 with losing. Given a well-founded win or lose game $G$ we call a strategy $s_{i}$ of player $i$ a winning strategy if $\forall s_{-i} \in S_{-i} p_{i}\left(\right.$ leaf $\left.\left(s_{i}, s_{-i}\right)\right)=1$. Below we denote the (possibly empty) set of winning strategies of player $i$ in $G$ by $\operatorname{win}_{i}(G)$.

A classic result, attributed to Zermelo [23], implies that in finite win or lose games one of the players has a winning strategy. This result also holds for arbitrary well-founded games.

Theorem 14 Let $G$ be a well-founded win or lose game. For all players $i$ we have $\operatorname{win}_{i}(G) \neq \emptyset$ iff $\operatorname{win}_{-i}(G)=\emptyset$.

Proof. We have the following sequences of equivalences, where $i=\operatorname{turn}(v)$ :

$$
s_{i} \in \operatorname{win}_{i}(G)
$$

iff $\quad\left\{\right.$ the definition of $\left.\operatorname{win}_{i}(G)\right\}$

for all $s_{-i} \in S_{-i}, p_{i}\left(\right.$ leaf $\left.\left(s_{i}, s_{-i}\right)\right)=1$

iff $\quad\{i=\operatorname{turn}(v)\}$

for all $s_{-i}^{w} \in S_{-i}^{w}, p_{i}\left(\operatorname{leaf}\left(s_{i}^{w}, s_{-i}^{w}\right)\right)=1$, where $w=s_{i}(v)$

iff $\quad$ definition of a winning strategy

$s_{i}^{w} \in \operatorname{win}_{i}\left(G^{w}\right)$, where $w=s_{i}(v)$.

and

$s_{-i} \in \operatorname{win}_{-i}(G)$

iff $\quad\left\{\right.$ the definition of $\left.\operatorname{win}_{-i}(G)\right\}$

for all $s_{i} \in S_{i}, p_{-i}\left(\operatorname{leaf}\left(s_{i}, s_{-i}\right)\right)=1$

iff $\quad\{i=\operatorname{turn}(v)\}$

for all $w \in C(v)$ and $s_{i}^{w} \in S_{i}^{w}, p_{-i}\left(\right.$ leaf $\left.\left(s_{i}^{w}, s_{-i}^{w}\right)\right)=1$

iff $\quad$ definition of a winning strategy

for all $w \in C(v), s_{-i}^{w} \in \operatorname{win}_{-i}\left(G^{w}\right)$. 
We now prove the claim by induction on the rank of the game tree. For game trees of rank 0 the claim clearly holds. Suppose that it holds for all well-founded win or lose games with game trees of rank smaller than some ordinal $\alpha>0$ and consider a win or lose game $G$ with the well-founded game tree of rank $\alpha$ and rooted at $v$. Let $i=\operatorname{turn}(v)$.

By the induction hypothesis for all $w \in C(v), \operatorname{win}_{i}\left(G^{w}\right) \neq \emptyset$ iff $\operatorname{win}_{-i}\left(G^{w}\right)=\emptyset$, so the above equivalences imply the following string of equivalences:

$\operatorname{win}_{i}(G) \neq \emptyset$ iff for some $w \in C(v), \operatorname{win}_{i}\left(G^{w}\right) \neq \emptyset$ iff for some $w \in C(v), \operatorname{win}_{-i}^{w}(G)=\emptyset$ iff $\operatorname{win}_{-i}(G)=\emptyset$

and hence also $\operatorname{win}_{-i}(G) \neq \emptyset$ iff $\operatorname{win}_{i}(G)=\emptyset$.

From Corollary 9 we know that every well-founded win or lose game has a subgame perfect equilibrium, thus in particular a Nash equilibrium. The following result clarifies the relation between Nash equilibria and winning strategies. We denote the set of Nash equilibria in an extensive game $G$ by $N E(G)$.

Corollary 15 Consider a well-founded win or lose game $G$. For some player $i, N E(G)=$ win $_{i}(G) \times S_{-i}$.

Proof. By Theorem $14 \operatorname{win}_{1}(G) \neq \emptyset$ or $\operatorname{win}_{2}(G) \neq \emptyset$. Suppose without loss of generality that $\operatorname{win}_{1}(G) \neq$ $\emptyset$.

$(\Rightarrow)$ Let $\left(s_{1}, s_{2}\right)$ be a Nash equilibrium and $t_{1}$ be a winning strategy for player 1 . Then we have $p_{1}\left(\right.$ leaf $\left.\left(s_{1}, s_{2}\right)\right) \geq p_{1}\left(\right.$ leaf $\left.\left(t_{1}, s_{2}\right)\right)=1$ and hence $p_{2}\left(\right.$ leaf $\left.\left(s_{1}, s_{2}\right)\right)=-1$. If $s_{1}$ is not a winning strategy for player 1 , then for some player 2 strategy $t_{2}$ we have $p_{1}$ (leaf $\left.\left(s_{1}, t_{2}\right)\right)=-1$, i.e., $p_{2}\left(\right.$ leaf $\left.\left(s_{1}, t_{2}\right)\right)=1>$ $p_{2}\left(\right.$ leaf $\left.\left(s_{1}, s_{2}\right)\right)$, which contradicts the fact that $\left(s_{1}, s_{2}\right)$ is a Nash equilibrium. So $N E(G) \subseteq \operatorname{win}_{1}(G) \times S_{2}$.

$(\Leftarrow)$ Take a winning strategy $s_{1}$ for player 1 . Then for all strategies $t_{1}$ of player 1 and $s_{2}$ and $t_{2}$ of player 2 ,

$$
p_{1}\left(\text { leaf }\left(t_{1}, s_{2}\right)\right) \leq p_{1}\left(\text { leaf }\left(s_{1}, s_{2}\right)\right)=p_{1}\left(\text { leaf }\left(s_{1}, t_{2}\right)\right) .
$$

So $\left(s_{1}, s_{2}\right)$ is a Nash equilibrium. Hence $\operatorname{win}_{1}(G) \times S_{2} \subseteq N E(G)$.

In general the sets of subgame perfect equilibria and Nash equilibria differ, so we cannot replace in the above result $N E(G)$ by $\operatorname{SPE}(G)$. However, the above corollary directly implies the following characterization of subgame perfect equilibria.

Corollary 16 Let $G$ be a well-founded win or lose game on a game tree $(V, E)$ with the set of leaves $Z$. Then $\operatorname{SPE}(G)=\left\{s \in S \mid \forall w \in V \backslash Z \exists i\left[s^{w} \in \operatorname{win}_{i}\left(G^{w}\right) \times S_{-i}^{w}\right]\right\}$.

It is easy to see that one cannot reverse here the order of the quantifiers.

We now consider a related class of games often called chess-like games. These are two-player wellfounded extensive games in which the only possible outcomes are $(1,-1),(0,0)$, and $(-1,1)$, with 0 interpreted as a draw. We say that a strategy $s_{i}$ of player $i$ in such a game guarantees him at least a draw if

$$
\forall s_{-i} \in S_{-i} p_{i}\left(\text { leaf }\left(s_{i}, s_{-i}\right)\right) \geq 0,
$$

and denote the (possibly empty) set of such strategies by $\operatorname{draw}_{i}(G)$.

We now prove the following result for well-founded chess-like games. The set $\operatorname{win}_{i}(G)$ is defined as above.

Theorem 17 In every well-founded chess-like game G

$$
\operatorname{win}_{1}(G) \neq \emptyset \operatorname{or}_{\operatorname{win}_{2}}(G) \neq \emptyset \text { or }\left(\operatorname{draw}_{1}(G) \neq \emptyset \text { and } \operatorname{draw}_{2}(G) \neq \emptyset\right) .
$$


It states that in every chess-like game either one of the players has a winning strategy or each player has a strategy that guarantees him at least a draw. These three alternatives are mutually exclusive, since for all $i \in\{1,2\}, \operatorname{win}_{i}(G) \neq \emptyset$ implies both $\operatorname{win}_{-i}(G)=\emptyset$ and $\operatorname{draw}_{-i}(G)=\emptyset$.

Proof. We introduce the following abbreviations:

- $A$ for $\operatorname{win}_{1}(G) \neq \emptyset$,

- $B$ for $\operatorname{draw}_{2}(G) \neq \emptyset$,

- $C$ for $\operatorname{win}_{2}(G) \neq \emptyset$,

- $D$ for $\operatorname{draw}_{1}(G) \neq \emptyset$.

Let $G_{1}$ and $G_{2}$ be the modifications of $G$ in which each outcome $(0,0)$ is replaced for $G_{1}$ by $(-1,1)$ and for $G_{2}$ by $(1,-1)$. Then $\operatorname{win}_{1}\left(G_{1}\right)=\operatorname{win}_{1}(G), \operatorname{win}_{2}\left(G_{1}\right)=\operatorname{draw}_{2}(G), \operatorname{win}_{1}\left(G_{2}\right)=\operatorname{draw}_{1}(G)$, and $\operatorname{win}_{2}\left(G_{2}\right)=\operatorname{win}_{2}(G)$.

Hence by Theorem 14 applied to the games $G_{1}$ and $G_{2}$ we have $A \vee B$ and $C \vee D$, so $(A \wedge C) \vee(A \wedge$ $D) \vee(B \wedge C) \vee(B \wedge D)$, which implies $A \vee C \vee(B \wedge D)$, since $\neg(A \wedge C),(A \wedge D) \equiv A$, and $(B \wedge C) \equiv C$.

For finite games, the above result is formulated in [22, page 125]. The proof first uses backward induction (apparently the first use of it in the literature on game theory) to establish the existence of a Nash equilibrium. Subsequently, (what is now called) the Minimax theorem is invoked to conclude that the payoff to the first player (and hence the second, as well) in any Nash equilibrium is unique. Finally, it is observed that each possible payoff value corresponds to one of the three disjuncts in the above theorem. The above theorem clarifies that this result holds for well-founded games as well, and that it can be proved in a simple way, without the use of backward induction.

In [6] a proof of this result is provided for chess-like games in which infinite plays, interpreted as draw, are allowed. The proof does not rely on backward induction and is also valid for well-founded chess-like games.

Corollary 18 Consider a well-founded chess-like game G. For some player $i$

$$
N E(G)=\operatorname{win}_{i}(G) \times S_{-i} \operatorname{or~NE}_{(G)}=\operatorname{draw}_{i}(G) \times \operatorname{draw}_{-i}(G) .
$$

Proof. Consider the games $G_{1}$ and $G_{2}$ from the proof of Theorem 17 . We noticed there that $\operatorname{win}_{1}\left(G_{1}\right)=$ $\operatorname{win}_{1}(G), \operatorname{win}_{2}\left(G_{1}\right)=\operatorname{draw}_{2}(G), \operatorname{win}_{1}\left(G_{2}\right)=\operatorname{draw}_{1}(G)$, and $\operatorname{win}_{2}\left(G_{2}\right)=\operatorname{win}_{2}(G)$.

So if $\operatorname{win}_{1}(G) \neq \emptyset$, then by Corollary 15 applied to the game $G_{1}$ we get $N E\left(G_{1}\right)=\operatorname{win}_{1}(G) \times S_{2}$, and if $\operatorname{win}_{2}(G) \neq \emptyset$, then by Corollary 15 applied to the game $G_{2}$ we get $N E\left(G_{2}\right)=S_{1} \times \operatorname{win}_{2}(G)$.

Suppose now that for both players $i, \operatorname{win}_{i}(G)=\emptyset$. Then both $\operatorname{win}_{1}\left(G_{1}\right)=\emptyset$ and $\operatorname{win}_{2}\left(G_{2}\right)=\emptyset$, so by Corollary 15 applied to the games $G_{2}$ and $G_{1}$ we get both $N E\left(G_{2}\right)=\operatorname{draw}_{1}(G) \times S_{2}$ and $N E\left(G_{1}\right)=$ $S_{1} \times \operatorname{draw}_{2}(G)$. This implies $N E\left(G_{1}\right) \cap N E\left(G_{2}\right)=\operatorname{draw}_{1}(G) \times \operatorname{draw}_{2}(G)$.

Further, it is easy to see that $N E(G) \subseteq N E\left(G_{1}\right)$ and $N E(G) \subseteq N E\left(G_{2}\right)$. Thus we have established that for some player $i$

$$
N E(G) \subseteq \operatorname{win}_{i}(G) \times S_{-i} \text { or } N E(G) \subseteq \operatorname{draw}_{i}(G) \times \operatorname{draw}_{-i}(G) .
$$

To complete the proof, let $p_{i}$ denote the payoff function of player $i$ in the game $G$. Suppose there exists a player $i$ such that $\operatorname{win}_{i}(G) \neq \emptyset$ and let $s \in \operatorname{win}_{i}(G) \times S_{-i}$. By the definition of $\operatorname{win}_{i}(G)$ for all $s_{-i}^{\prime}$ we have $p_{i}\left(\right.$ leaf $\left.\left(s_{i}, s_{-i}^{\prime}\right)\right)=1$. Hence, since $G$ is a zero-sum game, for all $s_{-i}^{\prime}$ we have $p_{-i}\left(\right.$ leaf $\left.\left(s_{i}, s_{-i}^{\prime}\right)\right)=-1$. 
Since 1 is a maximum payoff for all $s_{i}^{\prime}$ we also have $p_{i}(\operatorname{leaf}(s)) \geq p_{i}\left(\operatorname{lea} f\left(s_{i}^{\prime}, s_{-i}\right)\right)$. This shows that $s$ is a Nash equilibrium of $G$.

Suppose now that for all players $i, \operatorname{win}_{i}(G)=\emptyset$. Fix some $i \in\{1,2\}$ and let $s \in \operatorname{draw}_{i}(G) \times d r a w_{-i}(G)$. By the definition of the sets $\operatorname{draw}_{i}(G)$

- for all $s_{-i}^{\prime}, p_{i}\left(\operatorname{leaf}\left(s_{i}, s_{-i}^{\prime}\right)\right) \geq 0$ and

- for all $s_{i}^{\prime}, p_{-i}\left(\right.$ leaf $\left.\left(s_{i}^{\prime}, s_{-i}\right)\right) \geq 0$.

Hence, since $G$ is a zero-sum game, $p($ lea $f(s))=(0,0)$ and

- for all $s_{-i}^{\prime}, p_{-i}\left(\right.$ leaf $\left.\left(s_{i}, s_{-i}^{\prime}\right)\right) \leq 0$ and

- for all $s_{i}^{\prime}, p_{i}\left(\right.$ leaf $\left.\left(s_{i}^{\prime}, s_{-i}\right)\right) \leq 0$.

This means that $s$ is a Nash equilibrium of $G$.

Corollary 19 Consider a well-founded chess-like game $G$ on a game tree $(V, E)$ with the set of leaves $Z$. Then

$$
\operatorname{SPE}(G)=\left\{s \in S \mid \forall w \in V \backslash Z \exists i\left[s^{w} \in\left(\operatorname{win}_{i}\left(G^{w}\right) \times S_{-i}^{w}\right) \cup\left(\operatorname{draw}_{i}\left(G^{w}\right) \times \operatorname{draw}_{-i}\left(G^{w}\right)\right)\right]\right\} .
$$

\section{Conclusions}

In this paper we studied well-founded extensive games with perfect information. We focused on the existence and structural characterization of the sets of subgame perfect equilibria. We also provided such characterizations for two classes of two-player zero-sum games: win or lose games and chess-like games. It will be interesting to consider in this setting other notions and solution concepts that have been well-studied in finite games.

One of them is weak dominance. For finite games, its relation to backward induction was studied in [14]. The authors showed that for finite game that satisfy the TDI condition from Section 3 the elimination of weakly dominated strategies is order independent and is guaranteed to solve the game. The author of [6, 7] studied zero-sum extensive games and showed that every such game with finitely many outcomes can be solved by iterated elimination of weakly dominated strategies.

The definition of weak dominance applies to well-founded extensive games, as well, but the resulting dynamics may be different. For instance, it is possible that the iterated elimination of weakly dominated strategies can then result in empty strategy sets for all or for some players. Also, it may happen that the elimination process has to be iterated over ordinals larger than $\omega$. It would be interesting to identify subclasses of well-founded extensive games which can be solved by the iterated elimination of weakly dominated strategies and for which it is order independent.

Another direction is a study of the dynamics of strategy improvement in terms of best (or better) response updates. For finite extensive games, the relation between the improvement dynamics and Nash equilibria was analyzed in [12, 4]. For restricted classes of infinite games of perfect information, improvement dynamics were studied in [3, 18]. It is an interesting question how the improvement dynamics and Nash and subgame perfect equilibria relate in well-founded extensive games.

\section{Acknowledgements}

We would like to thank the reviewers and Marcin Dziubiński for helpful comments. The second author was partially supported by the grant MTR/2018/001244. 


\section{References}

[1] C. Alós-Ferrer \& K. Ritzberger (2016): The Theory of Extensive Form Games. Springer, doi:10.1007/978-3662-49944-3.

[2] P. Battigalli (1997): On rationalizability in extensive games. Journal of Economic Theory 74, pp. 40-61, doi $10.1006 /$ jeth. 1996.2252

[3] E. Boros, K. Elbassioni, V. Gurvich \& K. Makino (2012): On Nash equilibria and improvement cycles in pure positional strategies for Chess-like and Backgammon-like n-person games. Discrete Mathematics 312(4), pp. 772-788, doi:10.1016/j.disc.2011.11.011.

[4] T. Brihaye, G. Geeraerts, M. Hallet \& S. Le Roux (2017): Dynamics and Coalitions in Sequential Games. In: Proc. 8th International Symposium on Games, Automata, Logics and Formal Verification, p. 136-150, doi $10.4204 /$ EPTCS.256.10.

[5] M. Escardo \& P. Oliva (2012): Computing Nash Equilibria of Unbounded Games. In: Turing-100. The Alan Turing Centenary, EPiC Series in Computing 10, pp. 53-65, doi $10.29007 / 1 \mathrm{wpl}$.

[6] C. Ewerhart (2002): Backward Induction and the Game-Theoretic Analysis of Chess. Games and Economic Behaviour 39, pp. 206-214, doi 10.1006/game.2001.0900.

[7] C. Ewerhart (2002): Iterated Weak Dominance in Strictly Competitive Games of Perfect Information. Journal of Economic Theory 107(2), pp. 474-482, doi:10.1006/jeth.2001.2958.

[8] D. Fudenberg \& D. Levine (1983): Subgame-perfect equilibria of finite and infinite-horizon games. Journal of Economic Theory 31(2), pp. 251-268, doi 10.1016/0022-0531(83)90076-5

[9] M.O. Jackson \& S. Wilkie (2005): Endogenous Games and Mechanisms: Side Payments Among Players. Review of Economic Studies 72, p. 543-566, doi:10.1111/j.1467-937X.2005.00342.x

[10] M.M. Kaminski (2019): Generalized Backward Induction: Justification for a Folk Algorithm. Games 34(3), doi $10.3390 / \mathrm{g} 10030034$.

[11] D. König (1927): Über eine Schlußweise aus dem Endlichen ins Unendliche. Acta Litt. Ac. Sci. 3, pp. $121-130$.

[12] N.S. Kukushkin (2002): Perfect information and potential games. Games and Economic Behavior 38(2), pp. 306-317, doi:10.1006/game.2001.0859.

[13] G.J. Mailath \& L. Samuelson (2006): Repeated Games and Reputation: Long-Run Relationships. Oxford University Press, doi: 10.1093/acprof:oso/9780195300796.001.0001

[14] L.M. Marx \& J.M. Swinkels (1997): Order Independence for Iterated Weak Dominance. Games and Economic Behaviour 18, pp. 219-245, doi 10.1006/game.1997.0525.

[15] M.J. Osborne \& A. Rubinstein (1994): A Course in Game Theory. The MIT Press.

[16] K. Ritzberger (2001): Foundations of Non-cooperative Game Theory. Oxford University Press, Oxford, UK.

[17] J.C. Rochet (1980): Selection on an Unique Equilibrium Value for Extensive Games with Perfect Information. Cahiers de mathématiques de la décision, Université Paris IX-Dauphine.

[18] S. Le Roux \& A. Pauly (2020): A Semi-Potential for Finite and Infinite Games in Extensive Form. Dynamic Games and Applications 10, pp. 120-144, doi:10.1007/s13235-019-00301-7

[19] U. Schwalbe \& P. Walker (2001): Zermelo and the Early History of Game Theory. Games and Economic Behavior 34(1), pp. 123-137, doi:10.1006/game.2000.0794.

[20] R. Selten (1965): Spieltheoretische Behandlung eines Oligopolmodells mit Nachfrageträgheit. Zeitschrift für die gesamte Staatswisenschaft 121, pp. 301-324 and 667-689.

[21] A.S. Troelstra \& D. van Dalen (1988): Constructivism in Mathematics an Introduction (Volume 1). Studies in Logic and the Foundations of Mathematics 121, Elsevier, doi 10.1016/S0049-237X(09)70523-3.

[22] J. von Neumann \& O. Morgenstern (2004): Theory of Games and Economic Behavior (60th Anniversary Commemorative Edition). Princeton Classic Editions, Princeton University Press. 
[23] E. Zermelo (1913): Über eine Anwendung der Mengenlehre auf die Theoriedes Schachspiels. In: Proc. of The Fifth International Congress of Mathematicians, Cambridge University Press, pp. 501-504.

\section{Appendix}

Lemma 6 Let $G$ be a well-founded extensive game over the game tree T. A joint strategy sis a subgame perfect equilibrium in $G$ iff for all non-leaf nodes $u$ in $T$ and all $y \in C(u)$

- $p_{i}\left(\right.$ leaf $\left.\left(s^{x}\right)\right) \geq p_{i}\left(\right.$ leaf $\left.\left(s^{y}\right)\right)$, where $i=\operatorname{turn}(u)$ and $s_{i}(u)=x$.

\section{Proof.}

$(\Rightarrow)$ Suppose $s$ is a subgame perfect equilibrium in $G$. Consider a non-leaf node $u$ in $T$. Let $i=\operatorname{turn}(u)$, $x=s_{i}(u)$ and take some $y \in C(u)$. Let $t_{i}^{u}$ be the strategy obtained from $s_{i}^{u}$ by assigning the node $y$ to $u$.

We now have $p_{i}\left(\right.$ leaf $\left.\left(s^{x}\right)\right)=p_{i}\left(\right.$ leaf $\left.\left(s^{u}\right)\right) \geq p_{i}\left(\operatorname{leaf}\left(t_{i}^{u}, s_{-i}^{u}\right)\right)=p_{i}\left(\operatorname{leaf}\left(s^{y}\right)\right)$, where the inequality holds by since $s^{u}$ a Nash equilibrium in $G^{u}$.

$(\Leftarrow)$ We proceed by induction on the rank of the game tree of $G$. For game trees of rank 0 the induction hypothesis is vacuously true. Suppose the claim holds for all well-founded extensive games whose game tree is of rank smaller than some ordinal $\alpha>0$. Consider a well-founded game $G$ over a game tree $T$ of rank $\alpha$ with the root $v$.

Consider any node $u$ in $T$ such that $u \neq v$. (Since $\alpha>0$, such a node $u$ exists.) Then $\operatorname{rank}\left(T^{u}\right)$ is smaller than $\alpha$ and for all nodes $w$ in $T^{u}$ we have $\left(s^{u}\right)^{w}=s^{w}$. By the induction hypothesis $s^{u}$ is a subgame perfect equilibrium in $G^{u}$, so a fortiori it is a Nash equilibrium in $G^{u}$. It remains to prove that $s$ is a Nash equilibrium in $G$.

Suppose not. Then there exists player $i$ and $t_{i} \in S_{i}$ such that for $t=\left(t_{i}, s_{-i}\right)$ we have $p_{i}($ leaf $(s))<$ $p_{i}($ leaf $(t))$. Recall that every joint strategy $s^{\prime}$ in $G$ defines a rooted path play $\left(s^{\prime}\right)$ in $T$. By the definition of $t$ these paths differ for $s$ and $t$ at a node at which player $i$ moves. So for some non-leaf node $u$ in $G$ with $\operatorname{turn}(u)=i$ we have $\operatorname{play}(s)=\sigma u x \pi_{1}$ and $\operatorname{play}(t)=\sigma u y \pi_{2}$, where $\sigma, \pi_{1}$ and $\pi_{2}$ are possibly empty sequences of nodes and $x \neq y$. So $s_{i}(u)=x$ and $t_{i}(u)=y$.

Case 1. $s^{y} \neq t^{y}$.

Take the first, starting from the root, non-leaf node $w$ in $T^{y}$ such that $s_{i}(w) \neq t_{i}(w)$. We have $p_{i}(\operatorname{leaf}(s))=p_{i}\left(\operatorname{leaf}\left(s^{x}\right)\right) \geq p_{i}\left(\right.$ leaf $\left.\left(s^{y}\right)\right)=p_{i}\left(\operatorname{leaf}\left(s^{w}\right)\right) \geq p_{i}\left(\right.$ leaf $\left.\left(t^{w}\right)\right)=p_{i}($ leaf $(t))$, where the first inequality holds by the assumptions for the considered implication for the node $v$ and the second by the fact that $s^{w}$ is a Nash equilibrium. So we get a contradiction.

Case 2. $s^{y}=t^{y}$.

We have $p_{i}\left(\right.$ leaf $\left.\left(s^{x}\right)\right)=p_{i}($ leaf $(s))<p_{i}($ leaf $(t))=p_{i}\left(\right.$ leaf $\left.\left(t^{y}\right)\right)=p_{i}\left(\right.$ leaf $\left.\left(s^{y}\right)\right)$. But given that $s_{i}(u)=$ $x$ this contradicts the assumption for the node $u$.

This concludes the proof. 